ANNALES

POLONICI MATHEMATICI

99.1 (2010)

\title{
Fonctions biharmoniques adjointes
}

\author{
par Emmanuel P. Smyrnelis (Athens)
}

\begin{abstract}
The study of the equation $\left(L_{2} L_{1}\right)^{*} h=0$ or of the equivalent system $L_{2}^{*} h_{2}=-h_{1}, L_{1}^{*} h_{1}=0$, where $L_{j}(j=1,2)$ is a second order elliptic differential operator, leads us to the following general framework: Starting from a biharmonic space, for example the space of solutions $\left(u_{1}, u_{2}\right)$ of the system $L_{1} u_{1}=-u_{2}, L_{2} u_{2}=0, L_{j}(j=1,2)$ being elliptic or parabolic, and by means of its Green pairs, we construct the associated adjoint biharmonic space which is in duality with the initial one.
\end{abstract}

Introduction. L'étude des solutions de l'équation biharmonique $\Delta^{2} u$ $=0$ a été ramenée à celle des couples satisfaisant au système $\Delta u_{1}=-u_{2}$, $\Delta u_{2}=0$, dits couples biharmoniques. Cette approche est applicable à des équations du type $L_{2}\left(L_{1} u\right)=0$, où $L_{j}(j=1,2)$ est un opérateur différentiel linéaire du second ordre elliptique ou parabolique et conduit à l'introduction des espaces biharmoniques.

Des difficultés nouvelles surgissent : à la place de travailler avec les fonctions des espaces harmoniques, on travaille ici avec des couples de fonctions soumis en plus à la contrainte de compatibilité; et la vie nous enseigne que le comportement des composantes d'un couple ne rentre pas facilement dans un moule!

Dans le même ordre d'idées, pour étudier l'équation adjointe $\left(L_{2} L_{1}\right)^{*} h$ $=0$, on considérera le système $L_{1}^{*} h_{1}=0, L_{2}^{*} h_{2}=-h_{1}$.

Pour définir les couples biharmoniques adjoints, on pourra s'inspirer d'une propriété classique, d'après laquelle la fonction de Green d'ordre 2 de l'opérateur $L_{2} L_{1}$ devient, par échange de deux variables, la fonction de Green d'ordre 2 relative à l'opérateur adjoint $\left(L_{2} L_{1}\right)^{*}$.

Dans le cadre d'un espace biharmonique elliptique $(\Omega, \mathscr{H})$ et de ses espaces harmoniques associés $\left(\Omega, \mathscr{H}_{1}\right),\left(\Omega, \mathscr{H}_{2}\right)$, on note $p_{y}^{j}$ [resp. $\left.\left(w_{y}, p_{y}^{2}\right)\right]$ un $\mathscr{H}_{j}$-potentiel de support harmonique $\{y\}(j=1,2)$ [resp. un couple potentiel pur de support biharmonique $\{y\}]$.

2010 Mathematics Subject Classification: 31B30, 31D05.

Key words and phrases: adjoint biharmonic functions, adjoint Green's pairs, duality. 
Pour construire notre espace biharmonique adjoint, $y \mapsto\left(w_{y}(x), p_{y}^{1}(x)\right)$ devra être un couple potentiel adjoint dans $\Omega$ de support $\{x\}$, les $w_{y}$ et $p_{y}^{1}$ étant choisis convenablement.

On montre que les deux espaces biharmoniques sont en dualité. Pour cela, on définit d'abord le système de trois mesures $\rho_{y}^{\omega}, \sigma_{y}^{\omega}, \tau_{y}^{\omega}$, dual du système de mesures biharmoniques, portées aussi par $\partial \omega$ avec $\omega$ ouvert relativement compact, à l'aide de la dualité des espaces harmoniques associés, ainsi que les couples biharmoniques et surharmoniques adjoints; remarquons que les deux premières mesures sont relatives aux espaces harmoniques adjoints et la troisième est la mesure de couplage. Ensuite, on établit la dualité des couples de Green et des propriétés analogues à celles de l'espace initial. On étudie aussi l'espace bidual et l'on montre que les mesures biharmoniques** coïncident avec les mesures biharmoniques $\lambda_{x}^{\omega}, \mu_{x}^{\omega}, \nu_{x}^{\omega}$ et que les couples $\left(w_{x}^{*}, p_{x}^{1 *}\right)$ sont des couples potentiels adjoints purs de support $\{x\}$.

À la fin, on donne des applications : 1) On montre que les couples adjoints sont les solutions du système $L_{2}^{*} h_{2}=-h_{1}, L_{1}^{*} h_{1}=0$. 2) À l'aide des fonctions de Green des opérateurs $L_{j}(j=1,2)$, on exprime les couples de Green pour l'opérateur $L_{2} L_{1}$ et son adjoint. 3) On montre, d'une part, l'identité entre les ensembles polaires relatifs à l'opérateur composé et à son adjoint et les polaires au sens classique et, d'autre part, l'identité entre les pointsfrontières $L_{2} L_{1}$-réguliers, $\left(L_{2} L_{1}\right)^{*}$-réguliers et $\Delta$-réguliers.

L'intérêt de la dualité des espaces harmoniques et sa relation avec la dualité des processus de Markov par rapport à une mesure sont bien connus (voir [3], 15], 16], 21], 22], 25], 34]). De même qu'on peut, dans le cas classique, considérer l'opérateur adjoint par rapport à la mesure de Lebesgue ou une autre mesure, de même on peut remplacer le potentiel $p_{y}$ par $f(y) p_{y}$, où $f$ est une fonction strictement positive quelconque. Dans notre cas, on aura une première dualité concernant l'opérateur $L_{1}$, une seconde dualité sur l'opérateur $L_{2}$ et une autre sur l'opérateur composé $L_{2} L_{1}$, cette dernière étant soumise à la condition de compatibilité (voir remarque 2.4). Remarquons enfin que dans le cadre de la thèse de N. Bouleau ([6]), la dualité des processus de Markov correspondants présente un intérêt certain.

Pour les notions et notations non expliquées dans ce travail, voir [27.

Notons que ce travail se place dans le cadre d'une théorie locale. C'est seulement dans ce cadre qu'on examine la dualité des espaces considérés.

Signalons enfin que dans les références figurent certains travaux uniquement pour des raisons bibliographiques.

1. Rappels et quelques résultats préliminaires. Le cadre de cette partie sera un espace biharmonique elliptique fort avec $\Omega$ connexe. On suppose aussi la $j$-proportionnalité $(j=1,2)$. On désigne par $\mathscr{U}$ (resp. $\mathscr{U}_{c}$ ) 
l'ensemble des ouverts non vides (resp. des ouverts non vides relativement compacts) de $\Omega$. Soit $\mathscr{H}$ une application associant à chaque $U \in \mathscr{U}$ un sous-espace vectoriel de couples $\left(u_{1}, u_{2}\right)$ de $C(U) \times C(U)$, compatibles dans le sens que, si $u_{1}$ est nul dans un ouvert, $u_{2}$ y sera aussi. Les couples de $\mathscr{H}(U)$ seront dits biharmoniques dans $U$.

Un ensemble $\omega \in \mathscr{U}_{c}$ avec $\partial \omega \neq \emptyset$ est dit $\mathscr{H}$-régulier si :

(i) Le problème aux limites de Riquier a une solution biharmonique unique $\left(H_{1}^{\omega, f}, H_{2}^{\omega, f}\right)$ pour tout couple $f=\left(f_{1}, f_{2}\right) \in C(\partial \omega) \times C(\partial \omega)$.

(ii) $f_{j} \geq 0(j=1,2)$ implique $H_{1}^{\omega, f} \geq 0$ et $f_{2} \geq 0$ entraîne $H_{2}^{\omega, f} \geq 0$.

On aura ainsi, pour chaque $x \in \omega$, un unique système $\left(\lambda_{x}^{\omega}, \mu_{x}^{\omega}, \nu_{x}^{\omega}\right)$ de mesures positives de Radon sur $\partial \omega$ tel que $H_{1}^{\omega, f}(x)=\int f_{1} d \mu_{x}^{\omega}+\int f_{2} d \nu_{x}^{\omega}$, $H_{2}^{\omega, f}(x)=\int f_{2} d \lambda_{x}^{\omega}$.

Un couple de fonctions $\left(v_{1}, v_{2}\right)$ définies dans $U \in \mathscr{U}$ avec $v_{j}: U \rightarrow$ $(-\infty,+\infty]$ est dit hyperharmonique si les $v_{j}(j=1,2)$ sont semi-continues inférieurement et $v_{1}(x) \geq \int v_{1} d \mu_{x}^{\omega}+\int v_{2} d \nu_{x}^{\omega}, v_{2}(x) \geq \int v_{2} d \lambda_{x}^{\omega}$ pour tout $\omega$ ouvert-régulier $\subset \bar{\omega} \subset U$ et tout $x \in \omega$.

L'ensemble de ces couples sera noté $\mathscr{H}^{*}(U)\left(+\mathscr{H}^{*}(U)\right.$ s'il s'agit de couples positifs).

Si la fonction $v_{1}$ est finie sur un ensemble dense de $U$, le couple hyperharmonique $\left(v_{1}, v_{2}\right)$ sera dit surharmonique dans $U$.

$\mathscr{S}(U)$ désignera l'ensemble des couples surharmoniques dans $U\left(\mathscr{S}^{+}(U)\right.$ s'il s'agit des couples positifs).

Enfin, un couple $p=\left(p_{1}, p_{2}\right) \in \mathscr{S}^{+}(U)$ est dit couple potentiel (dans $U$ ) si $\left(h_{1}, h_{2}\right)=(0,0)$ est le seul couple biharmonique satisfaisant à $0 \leq h_{j} \leq p_{j}$ $(j=1,2)$.

$\mathscr{P}(U)$ est l'ensemble de tous les potentiels dans $U$.

Muni de quatre axiomes, I-IV, l'espace $(\Omega, \mathscr{H})$ est dit biharmonique (voir [27]). Un espace biharmonique est dit elliptique si, pour tout $x \in \Omega$ et tout $\omega$ ouvert $\mathscr{H}$-régulier contenant le point $x, \operatorname{supp}\left(\lambda_{x}^{\omega}\right)=\operatorname{supp}\left(\mu_{x}^{\omega}\right)=$ $\operatorname{supp}\left(\nu_{x}^{\omega}\right)=\partial \omega$; il est dit fort s'il existe un couple potentiel strictement positif dans $\Omega$.

À un espace biharmonique, on associe les deux espaces harmoniques sous-jacents $\left(\Omega, \mathscr{H}_{1}\right),\left(\Omega, \mathscr{H}_{2}\right)$, relatifs dans le cas classique aux solutions respectives de $L_{1} u_{1}=0, L_{2} u_{2}=0$.

On utilisera respectivement le préfixe 1 ou 2 pour les notions relatives aux espaces harmoniques précédents.

$\mathscr{H}_{j}^{*}(U), \mathscr{S}_{j}(U), \mathscr{P}_{j}(U)$ désigneront respectivement les ensembles de fonctions $j$-hyperharmoniques, $j$-surharmoniques et $j$-potentiels $(j=1,2)$ dans $U \in \mathscr{U}$. 
Le couple hyperharmonique (resp. surharmonique; potentiel) $\left(v_{1}, v_{2}\right)$ est dit pur dans $U$ si, étant donné $v_{2} \in{ }_{+} \mathscr{H}_{2}^{*}(U), v_{1}$ est la plus petite fonction $\geq 0$ telle que $\left(v_{1}, v_{2}\right) \in{ }_{+} \mathscr{H}^{*}(U)$ (resp. $\in \mathscr{S}^{+}(U) ; \in \mathscr{P}(U)$ ).

Le support $j$-harmonique (resp. biharmonique) d'une fonction $j$-hyperharmonique (resp. d'un couple hyperharmonique) est le plus petit fermé en dehors duquel la fonction (resp. le couple) est $j$-harmonique (resp. biharmonique) $(j=1,2)$.

Nous appelons couple de Green un couple potentiel pur de support biharmonique ponctuel. On sait que tout couple potentiel de support biharmonique ponctuel est égal à la somme d'un couple de Green et d'un couple $\left(p_{y}^{1}, 0\right)$, où $p_{y}^{1}$ est un 1-potentiel de support ponctuel ([32]).

Rappelons enfin que, si $\varphi$ est une fonction numérique dans un ouvert $U$, on définit sa régularisée $\widehat{\varphi}$ dans $U$ comme suit :

$$
\widehat{\varphi}(x)=\liminf _{y \in U, y \rightarrow x} \varphi(y) .
$$

DÉfinition 1.1. Un ouvert $\omega \in \mathscr{U}_{c}$ est dit $\mathscr{H}$-complètement déterminant ( $\mathscr{H}$-c.d.) si, pour tout couple potentiel $p=\left(p_{1}, p_{2}\right)$ biharmonique dans $\omega, P_{j}^{\complement \omega}=p_{j}$ dans $\Omega(j=1,2)$.

On reprend maintenant la définition 3.1 de [27] avec une base $\mathscr{A}$ d'ouverts $\in \mathscr{U}_{c}$ (sans supposer leur régularité). La proposition 3.2 ainsi que les propriétés qui suivent (partie III de [27]) restent valables.

Pour la définition et les propriétés des ouverts $j$-completèment déterminants $(j=1,2)$, voir [19].

Proposition 1.2. Soit $p_{2}$ un 2-potentiel dans $\Omega$ et $p_{1}^{\prime}$ la fonction hyperharmonique pure d'ordre 2 associée à $p_{2}$. Si, en un point $x_{0} \in \Omega, p_{1}^{\prime}\left(x_{0}\right)$ $<+\infty$, alors $\left(p_{1}^{\prime}, p_{2}\right)$ est un couple potentiel pur dans $\Omega$.

Démonstration. Voir définition 2.1 et corollaire 2.3 de 32 et corollaire 5.16 de [27.

Proposition 1.3. Soient $\left(u_{1}, u_{2}\right),\left(v_{1}, v_{2}\right)$ des couples hyperharmoniques purs dans $\Omega$ tels que $u_{2} \leq v_{2}$. Alors $v_{1}=u_{1}+s_{1}$, où $s_{1} \in{ }_{+} \mathscr{H}_{1}^{*}(\Omega)$.

Démonstration. On considère deux suites croissantes de couples potentiels purs finis continus $\left(p_{1}^{n}, p_{2}^{n}\right),\left(q_{1}^{n}, q_{2}^{n}\right)$ telles que $\left(u_{1}, u_{2}\right)=\left(\sup _{n} p_{1}^{n}\right.$, $\left.\sup _{n} p_{2}^{n}\right)$ et $\left(v_{1}, v_{2}\right)=\left(\sup _{n} q_{1}^{n}, \sup _{n} q_{2}^{n}\right)$ (lemme 10 de [28]).

On peut supposer que $q_{2}^{n} \geq p_{2}^{n}$, en prenant pour chaque $n$ la fonction $\inf \left(p_{2}^{n}, q_{2}^{n}\right)$ à la place de $p_{2}^{n}$ : En effet, si $u_{2}\left(x_{0}\right)=v_{2}\left(x_{0}\right)$ en un point $x_{0} \in \Omega$ et $\lambda \in \mathbb{R}$ tel que $\lambda<u_{2}\left(x_{0}\right)$, alors il existe $n_{0} \in \mathbb{N}$ tel que pour $n \geq n_{0}$, $\lambda<p_{2}^{n}\left(x_{0}\right) \leq u_{2}\left(x_{0}\right)$ et $\lambda<q_{2}^{n}\left(x_{0}\right) \leq v_{2}\left(x_{0}\right)$; d'où $\lambda<\inf \left(p_{2}^{n}\left(x_{0}\right), q_{2}^{n}\left(x_{0}\right)\right)$ $\leq u_{2}\left(x_{0}\right)$. D'autre part, si $u_{2}\left(x_{0}\right)<v_{2}\left(x_{0}\right)$ et $\lambda \in \mathbb{R}$ tel que $u_{2}\left(x_{0}\right)<\lambda$ $<v_{2}\left(x_{0}\right)$, alors il existe $m_{0} \in \mathbb{N}$ tel que pour $n \geq m_{0}, \lambda<q_{2}^{n}\left(x_{0}\right) \leq v_{2}\left(x_{0}\right)$ et $u_{2}\left(x_{0}\right)-\varepsilon<p_{2}^{n}\left(x_{0}\right) \leq u_{2}\left(x_{0}\right)(\varepsilon>0)$; d'où $p_{2}^{n}\left(x_{0}\right)<q_{2}^{n}\left(x_{0}\right)$. 
On sait que $\Gamma_{1} p_{1}^{n}=p_{2}^{n}, \Gamma_{1} q_{1}^{n}=q_{2}^{n}$ (théorème 11.9 de [27]); d'où $\Gamma_{1}\left(q_{1}^{n}-p_{1}^{n}\right)=q_{2}^{n}-p_{2}^{n} \geq 0$. Donc la fonction $q_{1}^{n}-p_{1}^{n}=s_{1}^{n}$ est 1-hyperharmonique positive car $q_{1}^{n} \geq p_{1}^{n}$ (théorème 11.3 de [27] et lemme 8 de [28]). Par conséquent, $q_{1}^{n}=p_{1}^{n}+s_{1}^{n}$ et, en passant à la limite, on trouve que $v_{1}=u_{1}+s_{1}$, où $s_{1} \in{ }_{+} \mathscr{H}_{1}^{*}(\Omega)$.

Lemme 1.4. Soit $p_{2}$ un 2-potentiel dans $\Omega$, harmonique dans un ouvert $\omega \in \mathscr{U}_{c}$. Alors, il existe une suite croissante de couples potentiels $\left(p_{1}^{n}, p_{2}^{n}\right)$ dans $\Omega$, biharmoniques dans $\omega$, telle que $\sup _{n} p_{2}^{n}=p_{2}$.

Démonstration. 1) S'il existe un 1-potentiel $p_{1}$ tel que $\left(p_{1}, p_{2}\right)$ soit un couple potentiel pur dans $\Omega$, alors, grâce à la proposition 2.8 de [32], c'est immédiat.

2) Supposons maintenant que le couple hyperharmonique $\left(+\infty, p_{2}\right)$ est un couple pur. Soient $\mu$ la mesure associée à $p_{2}$ et $\left(\omega_{n}\right)$ une suite croissante d'ouverts $\in \mathscr{U}_{c}$ telle que $\omega_{1}=\omega, \bar{\omega}_{n} \subset \omega_{n+1}$ et $\bigcup_{n=1}^{\infty} \omega_{n}=\Omega$. Soient encore $p_{2}^{\omega_{n}}$ la restriction spécifique de $p_{2}$ à $\omega_{n}$ et $\mu_{n}$ sa mesure associée. On voit que $\sup _{n} p_{2}^{\omega_{n}}=p_{2}$ (théorème 12.2 et pp. 461-463 de [19]); d'autre part, tous ces potentiels $p_{2}^{n}=p_{2}^{\omega_{n}}$ sont 2-harmoniques dans $\omega$.

On considère les couples $\left(p_{1}^{n}, p_{2}^{n}\right)=\left(\int w_{y} d \mu_{n}(y), \int p_{y}^{2} d \mu_{n}(y)\right)$. D'après le lemme 3.12 de [32] et la proposition 1.2, ces couples sont des couples potentiels purs dans $\Omega$ et, d' après la proposition 2.8 de [32], biharmoniques dans $\omega$.

THÉORÈme 1.5. Soit un ouvert $\omega \in \mathscr{U}_{c}$. Les conditions suivantes sont équivalentes :

(i) $\omega$ est $\mathscr{H}$-complètement déterminant.

(ii) $\omega$ est 1- et 2-complètement déterminant.

Démonstration. (i) $\Rightarrow$ (ii). On montre d'abord que $\omega$ est 2-c.d. : En effet, soit $p_{2}$ un 2-potentiel, harmonique dans $\omega$, et soit $p_{n}=\left(p_{1}^{n}, p_{2}^{n}\right)$ la suite de couples potentiels du lemme 1.4. D'après l'hypothèse, la réduite du couple $p_{n}$ sur $\complement \omega,\left(P_{n, 1}^{\complement \omega}, P_{n, 2}^{\complement} \omega\right)=\left(p_{1}^{n}, p_{2}^{n}\right)$ dans $\Omega$; d'où $P_{2}^{\complement \omega}=R_{p_{2}}^{\complement \omega}=p_{2}$ dans $\Omega$. Donc $\omega$ est 2 -complètement déterminant.

On verra maintenant que $\omega$ est aussi 1-c.d. : En effet, soit $p_{1}$ un 1potentiel, harmonique dans $\omega$. En considérant le couple potentiel $\left(p_{1}, 0\right)$, on obtient, grâce à l'hypothèse, $R_{p_{1}}^{\complement \omega}=p_{1}$ dans $\Omega$. Par conséquent, $\omega$ est 1-complètement déterminant.

(ii) $\Rightarrow($ i $)$. Soit $\left(p_{1}, p_{2}\right)$ un couple potentiel, biharmonique dans $\omega$ et soit $\left(v_{1}, v_{2}\right) \in \mathscr{S}^{+}(\Omega)$ avec $v_{j} \geq p_{j}$ dans $\complement \omega(j=1,2)$. Comme $v_{2} \in \mathscr{S}_{2}^{+}(\Omega)$ et que $p_{2}$ est un 2-potentiel, harmonique dans $\omega$, on a, grâce à l'hypothèse, $v_{2} \geq p_{2}$ dans $\Omega$. 
Considérons les couples purs $\left(p_{1}^{\prime}, p_{2}\right),\left(v_{1}^{\prime}, v_{2}\right)$. D'après la proposition 1.3, $v_{1}^{\prime} \succ p_{1}^{\prime}$ ou $v_{1}^{\prime}=p_{1}^{\prime}+t_{1}$ avec $t_{1} \in \mathscr{P}_{1}(\Omega)$. On sait que si $p_{2}$ est 2 -harmonique (1) dans $\omega,\left(p_{1}^{\prime}, p_{2}\right)$ est biharmonique dans $\omega$ (proposition 2.8 de [32]). Grâce au corollaire 11.4 de [27], dans $\omega: \Gamma_{1} p_{1}=p_{2}$ et $\Gamma_{1} p_{1}^{\prime}=p_{2}$, d'où $\Gamma_{1}\left(p_{1}-p_{1}^{\prime}\right)=0$. Donc, $p_{1}-p_{1}^{\prime}$ est 1-harmonique dans $\omega$. D'après la proposition 2.2 de [32] (voir aussi le lemme 2.7 de [30]), $p_{1}-p_{1}^{\prime}=r_{1}$ est un 1-potentiel dans $\Omega$; d'autre part $v_{1} \succ v_{1}^{\prime}$. On voit donc que $p_{1}-p_{1}^{\prime}=r_{1}$ est un 1-potentiel

harmonique dans $\omega$ et que $v_{1} \succ p_{1}^{\prime}$ ou $v_{1}-p_{1}^{\prime}=s_{1}$ avec $s_{1} \in \mathscr{S}_{1}^{+}(\Omega)$.

Comme $v_{1} \geq p_{1}$ dans $\complement \omega$ par hypothèse, on a $v_{1}-p_{1}^{\prime}=s_{1} \geq r_{1}=p_{1}-p_{1}^{\prime}$ dans $\complement \omega ; \omega$ étant un ouvert 1-c.d., il en résulte que $v_{1}-p_{1}^{\prime} \geq p_{1}-p_{1}^{\prime}$ dans $\Omega$, donc $v_{1} \geq p_{1}$ dans $\Omega$. D'où le résultat.

\section{Définition des couples biharmoniques adjoints}

Hypothèses. On se placera dans un espace biharmonique elliptique fort avec $\Omega$ connexe.

On supposera en plus pour les espaces harmoniques associés $\left(\Omega, \mathscr{H}_{1}\right)$, $\left(\Omega, \mathscr{H}_{2}\right)$ la proportionnalité des potentiels (dans $\Omega$ ) de support harmonique ponctuel et l'existence d'une base $\mathscr{B}$ d'ouverts de $\Omega$ formée de domaines, à la fois 1 - et 2 -complètement déterminants, donc $\mathscr{H}$-complètement déterminants d'après le théorème 1.5 .

Voici d'abord une propriété valable dans le cas classique :

Soit $\omega$ un ouvert de $\mathbb{R}^{n}$ admettant $G_{j}(x, y)$ comme fonction de Green relative à l'opérateur elliptique $L_{j}(j=1,2)$. La fonction de Green d'ordre 2, $N^{(2)}(x, y)=\int_{\omega} G_{1}(x, z) G_{2}(z, y) d z$, relative à l'opérateur $L_{2} L_{1}$, devient, par échange de deux variables, la fonction de Green d'ordre 2 relative à l'opérateur $\left(L_{2} L_{1}\right)^{*}, N^{*(2)}(x, y)=\int_{\omega} G_{2}^{*}(x, z) G_{1}^{*}(z, y) d z$, où $G_{j}^{*}(x, y)$ est la fonction de Green relative à l'opérateur $L_{j}^{*}$, lorsque toutes celles-ci existent.

Dans notre cadre, $y \mapsto\left(w_{y}(x), p_{y}^{1}(x)\right)$ devra être un couple potentiel adjoint dans $\Omega$ de support $\{x\}$. Il faut donc que les trois mesures adjointes $\rho_{y}^{\omega}, \sigma_{y}^{\omega}, \tau_{y}^{\omega}$ de l'ouvert $\omega \in \mathscr{U}_{c}$, au point $y \in \omega$, satisfassent aux conditions suivantes :

$$
\left\{\begin{array}{l}
\int w_{z}(x) d \sigma_{y}^{\omega}(z)+\int p_{z}^{1}(x) d \tau_{y}^{\omega}(z) \leq w_{y}(x), \\
\int p_{z}^{1}(x) d \rho_{y}^{\omega}(z) \leq p_{y}^{1}(x),
\end{array}\right.
$$

avec $=$ si $x \notin \bar{\omega}$ et $<$ si $x$ est dans la même composante connexe de $\omega$ que $y$; de plus, toutes ces trois mesures doivent être portées par $\partial \omega$.

$\rho_{y}^{\omega}$ sera donc la mesure 1-harmonique adjointe et $\sigma_{y}^{\omega}$ la mesure 2-harmonique adjointe; ces deux mesures sont définies par 


$$
\widehat{R}_{p_{y}^{1}}^{\mathrm{C}}(x)=\int p_{z}^{1}(x) d \rho_{y}^{\omega}(z), \quad \widehat{R}_{p_{y}^{2}}^{\mathrm{\omega}}(x)=\int p_{z}^{2}(x) d \sigma_{y}^{\omega}(z)
$$

([19, p. 537]).

Il nous reste à définir la mesure $\tau_{y}^{\omega}$.

D'après le lemme 3.12 de 32 et la proposition précédente 1.2, le couple $\left(\int w_{z}(x) d \sigma_{y}^{\omega}(z), \int p_{z}^{2}(x) d \sigma_{y}^{\omega}(z)\right)=\left(t_{1}, t_{2}\right)$ est un couple potentiel pur dans $\Omega$; d'autre part, comme $\left(w_{y}, p_{y}^{2}\right)$ est un couple pur et que $\int p_{z}^{2}(x) d \sigma_{y}^{\omega}(z) \leq p_{y}^{2}(x)$ ([19, p. 537]), on obtient

$$
w_{y}(x)=\int w_{z}(x) d \sigma_{y}^{\omega}(z)+q_{1}(x), \quad \text { où } q_{1} \in \mathscr{P}_{1}(\Omega) \text { (proposition 1.3) }
$$

( $q_{1}$ dépend naturellement de l'ouvert considéré $\omega$ et du point $y \in \omega$ ), d'où

$$
q_{1}(x)=w_{y}(x)-\int w_{z}(x) d \sigma_{y}^{\omega}(z) .
$$

Remarquons que le 1-potentiel $\int w_{z}(x) d \sigma_{y}^{\omega}(z)$ est fini partout : en effet, comme le couple $\left(t_{1}, t_{2}\right)$ est biharmonique dans $\Omega \backslash \partial \omega$, alors $t_{1}$ pourrait prendre la valeur $+\infty$ seulement pour $x \in \partial \omega$; or, il n'est pas possible que $t_{1}$ prenne la valeur $+\infty$ car alors le 1 -potentiel $q_{1}$ prendrait la valeur $-\infty$.

La première des conditions (1) est remplie par la balayée $\widehat{R}_{q_{1}}^{\complement \omega}$; d'où la définition de la mesure biharmonique adjointe $\tau_{y}^{\omega}$ :

DÉfinition 2.1. Étant donné un ouvert $\omega \in \mathscr{U}_{c}$ et $y \in \omega$, on désigne par $\tau_{y}^{\omega}$ la mesure positive de Radon définie par

$$
\widehat{R}_{q_{1}}^{\mathrm{C} \omega}(x)=\int p_{z}^{1}(x) d \tau_{y}^{\omega}(z) .
$$

On prouvera que cette mesure est portée par $\partial \omega$.

D'une part, on remarque que la balayée $\widehat{R}_{v_{1}}^{\mathrm{C} \omega}$ est 1-harmonique dans $\omega$ pour toute fonction $v_{1} \in \mathscr{S}_{1}^{+}(\Omega)$.

D'autre part, on a :

Lemme 2.2. Soient $\left(s_{1}, s_{2}\right),\left(t_{1}, t_{2}\right)$ deux couples surharmoniques purs dans $\Omega$ tels que $t_{2}=s_{2}$ dans un ouvert $U \in \mathscr{U}$. Alors, dans $U$, on $a$ $t_{1}=s_{1}+h_{1}$, où $h_{1}$ est une fonction 1-harmonique.

Démonstration. D'après le lemme 11.8 de [27], pour tout $\omega$ ouvert 1régulier $\subset \bar{\omega} \subset U$ et tout $x \in \omega$, on aura $t_{1}-s_{1}=H_{t_{1}}^{\omega}-H_{s_{1}}^{\omega}$ dans $\omega$; donc, la fonction $t_{1}-s_{1}=h_{1}$ est 1-harmonique dans $U$.

On sait déjà que les couples $\left(w_{y}, p_{y}^{2}\right),\left(\int w_{z}(x) d \sigma_{y}^{\omega}(z), \int p_{z}^{2}(x) d \sigma_{y}^{\omega}(z)\right)$ sont des couples potentiels purs dans $\Omega$. Comme $p_{y}^{2}(x)=\int p_{z}^{2}(x) d \sigma_{y}^{\omega}(z)$ pour $x \in \complement \bar{\omega}$, grâce au lemme précédent on a $w_{y}(x)=\int w_{z}(x) d \sigma_{y}^{\omega}(z)+h_{1}(x)$, où $h_{1}$ est une fonction 1-harmonique dans $\complement \bar{\omega}$.

De tout ce qui précède, on déduit que la mesure $\tau_{y}^{\omega}$ est portée par $\partial \omega$.

Dans tout ce qui suit, les notions et notations relatives à la théorie adjointe seront accompagnées soit d'une étoile soit du mot "adjoint(e)". 
DÉfinition 2.3. Un couple de fonctions réelles $\left(h_{2}^{*}, h_{1}^{*}\right)$, définies dans l'ouvert $\omega \in \mathscr{U}$, est dit biharmonique adjoint (ou biharmonique*) dans $\omega$ si

1) $h_{j}^{*}$ sont finies continues dans $\omega(j=1,2)$;

2) pour tout ouvert 1- et 2-c.d. $\delta \subset \bar{\delta} \subset \omega$ et tout $y \in \delta$,

$$
h_{2}^{*}(y)=\int h_{2}^{*} d \sigma_{y}^{\delta}+\int h_{1}^{*} d \tau_{y}^{\delta}, \quad h_{1}^{*}(y)=\int h_{1}^{*} d \rho_{y}^{\delta} .
$$

Remarque 2.4. Grâce au paragraphe "Propriétés de continuité" de 33 et au théorème 18.1 et à la proposition 22.1 de [19], on prend les $\left(w_{y}, p_{y}^{2}\right)$ dans une base compacte fixée $B$ du cône $\mathscr{S}^{+}(\Omega)$ de façon que le couple $y \mapsto\left(w_{y}(x), p_{y}^{1}(x)\right)$ soit continu dans $\Omega \backslash\{x\}$. Notons que

$$
B=\left\{\left(V_{1}, V_{2}\right) \in \mathscr{S}^{+}(\Omega): V_{1, f_{1}}\left(x_{1}\right)+V_{1, f_{2}}\left(x_{2}\right)+V_{2, f_{1}}\left(x_{1}\right)+V_{2, f_{2}}\left(x_{2}\right)=1\right\}
$$

définit aussi une base compacte $B_{1}$ du cône $\mathscr{S}_{1}^{+}(\Omega)$. (On a pris deux couples $\left(f_{1}, x_{1}\right),\left(f_{2}, x_{2}\right)$, où $\left.f_{i} \in \mathcal{K}^{+}\left(\bar{\Omega} \backslash\left\{x_{i}\right\}\right), x_{i} \in \Omega, i=1,2.\right)$ [Dans [33], p. 325, 2ème ligne, le démon de la typographie a remplacé le troisième signe + par le signe $=$.] Les couples biharmoniques adjoints qu'on a définis dépendent du choix du couple $\left(w_{y}, p_{y}^{1}\right)$. Comme deux couples $\left(w_{y}, p_{y}^{1}\right),\left(w_{y}^{\prime}, p_{y}^{1}\right)$ se déduisent l'un de l'autre par multiplication par un couple de fonctions $(\psi(y), \varphi(y))$, strictement positives et continues dans $\Omega$, il en sera de même pour les systèmes correspondants de couples biharmoniques adjoints. En effet, on considère le 2-potentiel $p_{y}^{\prime 2}=\psi(y) p_{y}^{2}$ et le couple pur correspondant $\left(\psi(y) w_{y}^{1}, \psi(y) p_{y}^{2}\right)$ ainsi que le 1-potentiel $p_{y}^{\prime 1}=\varphi(y) p_{y}^{1}$. Le nouveau système de mesures sera alors : $d \varrho_{y}^{\omega}(z)=\frac{1}{\varphi(z)} \varphi(y) d \varrho_{y}^{\omega}(z), d \sigma_{y}^{\prime \omega}(z)=\frac{1}{\psi(z)} \psi(y) d \sigma_{y}^{\omega}(z)$, $d \tau_{y}^{\prime \omega}(z)=\frac{1}{\varphi(z)} \psi(y) d \tau_{y}^{\omega}(z)$ (voir définition des mesures $\rho_{y}^{\omega}, \sigma_{y}^{\omega}, \tau_{y}^{\omega}$ ).

Exemples 2.5. 1) D'après ce qui précède, le couple $y \mapsto\left(w_{y}(x), p_{y}^{1}(x)\right)$ est biharmonique* dans $\Omega \backslash\{x\}$.

2) Si $\mu$ est une mesure de Radon positive, portée par un compact $K$ de $\Omega$, alors le couple $y \mapsto\left(\int w_{y}(x) d \mu(x), \int p_{y}^{1}(x) d \mu(x)\right)$ est biharmonique* dans $\Omega \backslash K$ : Soit $\omega$ un ouvert 1- et 2-c.d., $\bar{\omega} \subset \Omega \backslash K$, et $y \in \omega$; alors

$$
\begin{aligned}
\int\left[\int w_{z}\right. & (x) d \mu(x)] d \sigma_{y}^{\omega}(z)+\int\left[\int p_{z}^{1}(x) d \mu(x)\right] d \tau_{y}^{\omega}(z) \\
= & \int\left[\int w_{z}(x) d \mu(x)\right] d \sigma_{y}^{\omega}(z)+\int \widehat{R}_{q_{1}}^{\mathrm{C} \omega}(x) d \mu(x) \\
= & \int\left[\int w_{z}(x) d \mu(x)\right] d \sigma_{y}^{\omega}(z)+\int w_{y}(x) d \mu(x)-\int\left[\int w_{z}(x) d \mu(x)\right] d \sigma_{y}^{\omega}(z) \\
= & \int w_{y}(x) d \mu(x)
\end{aligned}
$$

car dans $\complement \bar{\omega}, \widehat{R}_{q_{1}}^{\mathrm{C} \omega}=q_{1}$. D'autre part,

$$
\int\left[\int p_{z}^{1}(x) d \mu(x)\right] d \rho_{y}^{\omega}(z)=\int \widehat{R}_{p_{y}^{1}}^{\complement \omega}(x) d \mu(x)=\int p_{y}^{1}(x) d \mu(x) .
$$


Proposition 2.6. Pour tout domaine 1-c.d. $\omega$ et tout $y \in \omega, \tau_{y}^{\omega} \neq 0$.

Démonstration. On sait que $\sigma_{y}^{\omega} \neq 0$ (lemme 29.3 de [19]) et que les couples $\left(w_{y}, p_{y}^{2}\right),\left(\int w_{z}(x) d \sigma_{y}^{\omega}(z), \int p_{z}^{2}(x) d \sigma_{y}^{\omega}(z)\right)$ sont surharmoniques purs (lemme 3.12 de [32] et proposition 1.2); d'autre part, $\int p_{z}^{2}(x) d \sigma_{y}^{\omega}(z) \leq p_{y}^{2}(x)$ dans $\Omega$ et $\int p_{z}^{2}(x) d \sigma_{y}^{\omega}(z)<p_{y}^{2}(x)$ dans $\omega$. D'après la proposition 1.3, $q_{1}(x)=$ $w_{y}(x)-\int w_{z}(x) d \sigma_{y}^{\omega}(z)>0$ dans $\Omega$. Soit $x_{1} \in \mathfrak{C} \bar{\omega}$; comme $\widehat{R}_{q_{1}}^{\mathrm{C \omega}}(x)=q_{1}(x)$ pour $x \in \complement \bar{\omega}$, alors $\int p_{z}^{1}\left(x_{1}\right) d \tau_{y}^{\omega}(z)>0$; or $z \mapsto p_{z}^{1}\left(x_{1}\right)$ est continue et $>0$ sur $\partial \omega$, donc $\tau_{y}^{\omega} \neq 0$.

Corollaire 2.7. Tout couple $\left(h_{2}^{*}, h_{1}^{*}\right)$ biharmonique* dans un ouvert $U \in \mathscr{U}$ est compatible.

Démonstration. Soit $U^{\prime} \in \mathscr{U}, U^{\prime} \subset U, h_{2}^{*}=0$ dans $U^{\prime}$. On montrera que $h_{1}^{*}=0$ dans $U^{\prime}$. Sinon, soit $x_{0} \in U^{\prime}$ où $h_{1}^{*}\left(x_{0}\right) \neq 0$, par exemple $h_{1}^{*}\left(x_{0}\right)>0$. Grâce à la continuité, il existe un voisinage $U_{x_{0}} \subset U^{\prime}$ où $h_{1}^{*}>0$; pour tout domaine 1- et 2-c.d. $\omega \subset \bar{\omega} \subset U_{x_{0}}$, on aura $h_{2}^{*}(y)=\int h_{2}^{*} d \sigma_{y}^{\omega}+\int h_{1}^{*} d \tau_{y}^{\omega}$, d'où $\int h_{1}^{*} d \tau_{y}^{\omega}=0$ pour tout $y \in \omega$. Contradiction, car $h_{1}^{*}>0$ sur $\partial \omega$ et $\tau_{y}^{\omega} \neq 0$ pour tout $y \in \omega$.

Proposition 2.8. Soit $\left(h_{2}^{*}, h_{1}^{*}\right)$ un couple biharmonique $\geq(0,0)$ dans un domaine $\omega$. Alors, on aura l'une des situations ci-après :

1) $h_{2}^{*}>0, h_{1}^{*}>0$;

2) $h_{2}^{*}>0, h_{1}^{*}=0$

3) $h_{2}^{*}=0, h_{1}^{*}=0$.

Démonstration. En raisonnant comme dans le théorème 29.2 de [19], on voit que d'une part $h_{2}^{*}>0$ ou $h_{2}^{*}=0$ dans $\omega$, et d'autre part $h_{1}^{*}>0$ ou $h_{1}^{*}=0$ dans $\omega$. Pour le couple $\left(h_{2}^{*}, h_{1}^{*}\right)$, on a donc quatre possibilités, dont l'une : $h_{2}^{*}=0, h_{1}^{*}>0$ est à rejeter en vertu du corollaire 2.7.

Proposition 2.9. Soit $\omega$ un ouvert 1 - et 2-c.d. Alors :

1) $\left\|\tau_{y}^{\omega}\right\| \rightarrow 0$ quand $y \rightarrow z_{0}, y \in \omega$ et $z_{0} \in \partial \omega$ (la convergence vague en découle).

2) Il existe une constante $M$ telle que $\left\|\tau_{y}^{\omega}\right\| \leq M$ pour tout $y \in \omega$.

3) $\left\|\tau_{y_{0}}^{\omega}\right\| \underset{\mathscr{F}_{y_{0}}}{\rightarrow} 0$, où $\mathscr{F}_{y_{0}}$ est le filtre des sections des ouverts 1 - et 2 -c.d. contenant le point $y_{0} \in \Omega$ (ordonnés par inclusion).

Démonstration. 1) Soit $x_{1} \in \complement \bar{\omega}$. On a

$$
w_{y}\left(x_{1}\right)-\int w_{z}\left(x_{1}\right) d \sigma_{y}^{\omega}(z)=\int p_{z}^{1}\left(x_{1}\right) d \tau_{y}^{\omega}(z) .
$$

Comme $\int w_{z}\left(x_{1}\right) d \sigma_{y}^{\omega}(z) \rightarrow w_{z_{0}}\left(x_{1}\right)$ (théorème 29.4 de [19]), alors $\int p_{z}^{1}\left(x_{1}\right) d \tau_{y}^{\omega}(z) \rightarrow 0$. (On rappelle que les fonctions $y \mapsto w_{y}(x), y \mapsto p_{y}^{1}(x)$ sont continues pour $y \neq x$.) La fonction $z \mapsto p_{z}^{1}\left(x_{1}\right)$ étant continue sur le 
compact $\partial \omega$, elle atteint son minimum $m>0$ sur $\partial \omega$. Donc $0<m \int d \tau_{y}^{\omega}(z) \leq$ $\int p_{z}^{1}\left(x_{1}\right) d \tau_{y}^{\omega}(z)$ et $\int d \tau_{y}^{\omega} \rightarrow 0$ quand $y \rightarrow x_{0}$.

2) Les fonctions $y \mapsto w_{y}\left(x_{1}\right)$ et $y \mapsto \int w_{z}\left(x_{1}\right) d \sigma_{y}^{\omega}(z)$ sont bornées sur $\bar{\omega}$ car continues (proposition 6 de 33 ; démonstration du théorème 29.4 de [19]). Par conséquent, il existe une constante $M$ telle que $\int p_{z}^{1}\left(x_{1}\right) d \tau_{y}^{\omega}(z)$ $\leq M$ pour tout $y \in \omega$.

On peut supposer $m>1$ (en multipliant au besoin par un $\lambda>0$ convenable, ce qui revient à considérer les couples $\left.\left(\lambda w_{y}, \lambda p_{y}^{1}\right)\right)$; donc $1 \leq p_{z}^{1}\left(x_{1}\right)$ pour tout $z \in \partial \omega$, d'où $\int d \tau_{y}^{\omega}(z) \leq \int p_{z}^{1}\left(x_{1}\right) d \tau_{y}^{\omega}(z) \leq M$.

3) Soit $x \in \Omega$. La fonction $y \mapsto w_{y}(x)$ étant 2-surharmonique* dans $\Omega$ (voir les inégalités (1) au début de cette partie et 2.5.1), il s'ensuit que $\int w_{z}(x) d \sigma_{y_{0}}^{\omega_{2}}(z) \leq \int w_{z}(x) d \sigma_{y_{0}}^{\omega_{1}}(z)$ si $\omega_{1}, \omega_{2}$ sont des ouverts 2-c.d. tels que $\bar{\omega}_{1} \subset \omega_{2}$, et $\sup _{\omega \backslash y_{0}} \int w_{z}(x) d \sigma_{y_{0}}^{\omega}(z)=w_{y_{0}}(z)$, où $\omega$ est un ouvert 2-c.d. contenant $y_{0}$. (Les ouverts $j$-c.d. et $j$-réguliers ${ }^{*}, j=1,2$, sont identiques : théorème 32.2 de [19].)

Comme $w_{y_{0}}(x)-\int w_{z}(x) d \sigma_{y_{0}}^{\omega}(z) \geq \int p_{z}^{1}(x) d \tau_{y_{0}}^{\omega}(z)$, on obtient $\int p_{z}^{1}(x) d \tau_{y_{0}}^{\omega}(z) \rightarrow 0$ lorsque $\omega \searrow y_{0}$. Supposons maintenant $x \neq y_{0}$. Soit $K$ un voisinage compact de $y_{0}$ ne contenant pas $x$. Sur $K$, la fonction $y \mapsto p_{y}^{1}(x)$ atteint son minimum $\mu>0$ qu'on peut supposer $\geq 1$. On obtient donc $\int d \tau_{y_{0}}^{\omega} \leq \int p_{z}^{1}(x) d \tau_{y_{0}}^{\omega}(z)$ pour tout $\omega 1$ - et 2 -c.d. $\subset \bar{\omega} \subset K$. D'où le résultat.

(Pour les propriétés des mesures $\sigma_{y}^{\omega}$ et $\rho_{y}^{\omega}$ se référer au cas harmonique.)

Proposition 2.10. Soit $\left(h_{2}^{*}, h_{1}^{*}\right)$ un couple biharmonique* dans un ouvert $\omega \in \mathscr{U}_{c}$, et tel que, pour tout $z \in \partial \omega$,

$$
\liminf _{\substack{x \rightarrow z \\ x \in \omega}} h_{j}^{*}(x) \geq 0 \quad(j=1,2) ;
$$

alors $\left(h_{2}^{*}, h_{1}^{*}\right) \geq(0,0)$ dans $\omega$.

Démonstration. $h_{1}^{*}$ étant 1-harmonique*, on obtient $h_{1}^{*} \geq 0$ dans $\omega$ (théorème 29.3 de [19]). Par conséquent, $h_{2}^{*}$ est 2 -surharmonique* et, grâce au principe du minimum adjoint (théorème 29.3 et lemme 11.2 de [19]), on trouve que $h_{2}^{*} \geq 0$ dans $\omega$.

DÉFINITION 2.11.

1) On dit que le couple $\left(v_{2}^{*}, v_{1}^{*}\right)$ est hyperharmonique adjoint (ou hyperharmonique $\left.{ }^{*}\right)$ dans $U \in \mathscr{U}$ si :

(a) les fonctions $v_{j}^{*}$ sont s.c.i. et $>-\infty$ dans $U(j=1,2)$,

(b) $v_{2}^{*}(y) \geq \int v_{2}^{*} d \sigma_{y}^{\omega}+\int v_{1}^{*} d \tau_{y}^{\omega}, v_{1}^{*}(y) \geq \int v_{1}^{*} d \rho_{y}^{\omega}$ pour tout ouvert 1et 2-c.d. $\omega \subset \bar{\omega} \subset U$ et tout $y \in \omega$.

2 ) On dit que le couple $\left(v_{2}^{*}, v_{1}^{*}\right)$ est surharmonique adjoint (ou surharmonique $e^{*}$ si, de plus, $v_{2}^{*}$ est finie sur un ensemble dense de $U$. 
Proposition 2.12. Pour toute mesure de Radon $\mu$ positive sur $\Omega$, le couple $\left(s_{2}^{*}(y), s_{1}^{*}(y)\right)=\left(\int w_{y}(u) d \mu(u), \int p_{y}^{1}(u) d \mu(u)\right)$ est hyperharmonique* dans $\Omega$. Si $\mu$ est portée par un compact $K$, alors $\left(s_{2}^{*}, s_{1}^{*}\right)$ est surharmonique* dans $\Omega$; en particulier, le couple $y \mapsto\left(w_{y}(x), p_{y}^{1}(x)\right)$, noté $\left(w_{x}^{*}(y), p_{x}^{1 *}(y)\right)$, est surharmonique* dans $\Omega$ (x fixé dans $\Omega$ ).

Démonstration. Le couple $\left(s_{2}^{*}, s_{1}^{*}\right) \geq(0,0)$ et les fonctions $s_{j}^{*}(j=1,2)$ sont s.c.i. dans $\Omega$ (proposition 6 de [33] et théorème 1, partie III, chap. I, $\S 3$, de [8]). D'autre part, pour tout ouvert 1- et 2-c.d. $\omega$ et $y \in \omega$,

$$
\begin{aligned}
\int s_{2}^{*} d \sigma_{y}^{\omega}+\int s_{1}^{*} d \tau_{y}^{\omega} & =\iint w_{z}(u) d \mu(u) d \sigma_{y}^{\omega}(z)+\iint p_{z}^{1}(u) d \mu(u) d \tau_{y}^{\omega}(z) \\
& =\int\left[\int w_{z}(u) d \sigma_{y}^{\omega}(z)+\int p_{z}^{1}(u) d \tau_{y}^{\omega}(z)\right] d \mu(u) .
\end{aligned}
$$

Or

$$
\int w_{z}(u) d \sigma_{y}^{\omega}(z)+\int p_{z}^{1}(u) d \tau_{y}^{\omega}(z) \leq w_{y}(u) .
$$

Par conséquent,

$$
\int s_{2}^{*} d \sigma_{y}^{\omega}+\int s_{1}^{*} d \tau_{y}^{\omega} \leq \int w_{y}(u) d \mu(u)=s_{2}^{*}(y) .
$$

D'autre part, on trouve aussi que $\int s_{1}^{*} d \rho_{y}^{\omega} \leq s_{1}^{*}(y)$ (cas harmonique).

Soit maintenant $\mu$ portée par un compact $K$. On remarque d'abord que $s_{2}^{*}$ est une fonction 2-hyperharmonique* dans $\Omega$ (car $s_{1}^{*} \geq 0$ ); alors, ou bien $s_{2}^{*}=+\infty$ ou bien $s_{2}^{*}$ est 2 -surharmonique* $([8])$. Mais, on a démontré dans l'exemple 2.5(2) que le couple $\left(s_{2}^{*}, s_{1}^{*}\right)$ est biharmonique* dans $\Omega \backslash K$; d'où le résultat.

THÉORÈme 2.13. Tout ouvert $\omega, 1$ - et 2-c.d., est régulier biharmonique*, le système biharmonique de mesures de $\omega$ au point $y \in \omega$ étant $\left(\rho_{y}^{\omega}, \sigma_{y}^{\omega}, \tau_{y}^{\omega}\right)$. La base $\mathscr{B}$ réalise donc l'axiome II pour les couples biharmoniques adjoints.

Démonstration. Soit $\left(f_{2}, f_{1}\right) \in C(\partial \omega) \times C(\partial \omega)$. On montrera que :

1) le couple

$$
\left(h_{2}^{*}(y), h_{1}^{*}(y)\right)=\left(\int f_{2} d \sigma_{y}^{\omega}+\int f_{1} d \tau_{y}^{\omega}, \int f_{1} d \rho_{y}^{\omega}\right)
$$

est biharmonique* dans $\omega$;

2) $\lim _{y \rightarrow z, y \in \omega} h_{j}^{*}(y)=f_{j}(z), \forall z \in \partial \omega, j=1,2$;

3) $\left(f_{2}, f_{1}\right) \geq(0,0)$ sur $\partial \omega$ implique $h_{2}^{*} \geq 0$ dans $\omega ; f_{1} \geq 0$ sur $\partial \omega$ implique $h_{1}^{*} \geq 0$ dans $\omega$.

1) Comme dans le théorème 29.4 de [19], on considère des fonctions $f_{1}^{x}(z)$ de la forme $\int p_{z}^{1}(u) d \mu_{x}^{U}(u), f_{2}^{x}(z)$ de la forme $\int p_{z}^{2}(u) d \lambda_{x}^{U}(u)$, où $U \in \mathscr{B}$, $x \in U, \mu_{x}^{U}$ est la mesure 1-harmonique, et $\lambda_{x}^{U}$ est la mesure 2-harmonique. On pose $\mu_{x}^{U}=\mu$ et $\lambda_{x}^{U}=\nu$; alors

$$
\int\left(\int p_{z}^{1}(u) d \mu(u)\right) d \tau_{y}^{\omega}(z)=\int \widehat{R}_{q_{1}}^{\mathrm{C} \omega}(u) d \mu(u)=\int q_{1}(u) d \mu^{\mathrm{C} \omega}(u)
$$


grâce à la propriété de la 1-balayée d'une mesure. La mesure $\mu^{\mathrm{C \omega}}$ est portée par le compact $(\complement \omega \cap \partial U) \cup \partial \omega$. Comme $q_{1}(u)=w_{y}(u)-\int w_{z}(u) d \sigma_{y}^{\omega}(z)$, on obtient

$$
\int q_{1}(u) d \mu^{\mathrm{C} \omega}(u)=\int w_{y}(u) d \mu^{\mathrm{C} \omega}(u)-\int\left(\int w_{z}(u) d \mu^{\mathrm{C} \omega}(u)\right) d \sigma_{y}^{\omega}(z) .
$$

Grâce à la proposition 2.12, le couple $\left(\int w_{y}(u) d \mu^{\mathrm{C} \omega}(u), \int p_{y}^{1}(u) d \mu^{\mathrm{C} \omega}(u)\right)$ est surharmonique* positif dans $\Omega$ et, d'après l'exemple 2.5(2), il est biharmonique* dans $\omega$; d'autre part, la fonction

$$
y \mapsto \int\left(\int w_{z}(u) d \mu^{\mathrm{C} \omega}(u)\right) d \sigma_{y}^{\omega}(z)
$$

est 2-harmonique* dans $\omega$. Finalement, le couple

$$
\left(\iint p_{z}^{2}(u) d \nu(u) d \sigma_{y}^{\omega}(z)+\iint p_{z}^{1}(u) d \mu(u) d \tau_{y}^{\omega}(z), \iint p_{z}^{1}(u) d \mu(u) d \rho_{y}^{\omega}(z)\right)
$$

est biharmonique* dans $\omega$ : En effet,

$$
\iint p_{z}^{1}(u) d \mu(u) d \rho_{y}^{\omega}(z)=\int \widehat{R}_{p_{y}^{1}}^{\mathrm{C}}(u) d \mu(u)=\int p_{y}^{1}(u) d \mu^{\mathrm{C} \omega}(u)
$$

à cause de la définition de la mesure $\rho_{y}^{\omega}$. On sait que le couple

$$
\left(\int\left(\int p_{z}^{1}(u) d \mu(u)\right) d \tau_{y}^{\omega}(z), \int\left(\int p_{z}^{1}(u) d \mu(u)\right) d \rho_{y}^{\omega}(z)\right)
$$

est biharmonique* dans $\omega$. D'autre part, grâce à la définition de la mesure $\sigma_{y}^{\omega}$, on obtient

$$
\iint p_{z}^{2}(u) d \nu(u) d \sigma_{y}^{\omega}(z)=\int \widehat{R}_{p_{y}^{2}}^{\mathrm{C}_{\omega}}(u) d \nu(u)=\int p_{y}^{2}(u) d \nu^{\mathrm{C} \omega}(u),
$$

la mesure $\nu^{\complement \omega}$ étant portée par le compact $(\complement \omega \cap \partial U) \cup \partial \omega$; donc, la fonction $y \mapsto \int p_{y}^{2}(u) d \nu^{\mathrm{C} \omega}(u)$ est 2 -harmonique* dans $\omega$. D'où le résultat.

2) Vu le théorème 29.4, le lemme 29.4 de [19] et la propriété 1) de la proposition $2.9,\left(\int f_{2} d \sigma_{y}^{\omega}+\int f_{1} d \tau_{y}^{\omega}, \int f_{1} d \rho_{y}^{\omega}\right)$ effectue un prolongement biharmonique* dans $\omega$ et continu dans $\bar{\omega}$ du couple $\left(f_{2}, f_{1}\right)$.

3) Les mesures $\rho_{y}^{\omega}, \sigma_{y}^{\omega}, \tau_{y}^{\omega}$ étant positives, on obtient immédiatement le résultat.

Le prolongement est unique en vertu de la proposition 2.10.

Proposition 2.14. Pour tout $\omega$ régulier biharmonique* connexe et tout $y \in \omega$, on a $T \tau_{y}^{\omega}=\partial \omega$ ( $T$ désignant le support).

Démonstration. En tenant compte de 2.6, 2.7, 2.8, on raisonne comme dans la démonstration de la proposition 2.5 de [27].

À noter que $T \rho_{y}^{\omega}=T \sigma_{y}^{\omega}=\partial \omega$ grâce à la définition de ces mesures.

3. Espace biharmonique adjoint. On maintient les mêmes hypothèses que dans la partie 2 . 
Proposition 3.1. Les conditions suivantes sont équivalentes:

(i) $\omega$ est un ouvert 1 - et 2-régulier*.

(ii) $\omega$ est un ouvert régulier biharmonique*.

Démonstration. (i) $\Rightarrow$ (ii). D'après le théorème 2.13 , tout ouvert 1- et 2c.d. est régulier biharmonique*. D'autre part, il y a identité entre les ouverts $j$-c.d. et les ouverts $j$-réguliers* $, j=1,2$ (théorème 32.2 de [19]).

(ii) $\Rightarrow(\mathrm{i})$. En effet, à tout couple $(f, 0)$, fini continu sur $\partial \omega$, correspond un seul couple $\left(h_{2}^{*}, 0\right)$ biharmonique* dans $\omega$ (théorème 2.13 ), donc une seule fonction $h_{2}^{*}, 2$-harmonique* dans $\omega$, telle que $\lim _{\omega \ni y \rightarrow z} h_{2}^{*}(y)=f(z)$ pour tout $z \in \partial \omega$. De même, à tout couple $(0, \phi)$, fini continu sur $\partial \omega$, correspond un seul couple $\left(h_{2}^{*}, h_{1}^{*}\right)$ biharmonique* dans $\omega$ tel que $\lim _{\omega \ni y \rightarrow z} h_{2}^{*}(y)=0$ et $\lim _{\omega \ni y \rightarrow z} h_{1}^{*}(y)=\phi(z)$ pour tout $z \in \partial \omega$.

Proposition 3.2. Il y a identité entre les ouverts $\mathscr{H}$-complètement déterminants et les ouverts réguliers biharmoniques*.

Démonstration. On sait qu'il y a identité :

- entre les ouverts 1- et 2-réguliers* et les ouverts réguliers biharmoniques* (proposition 3.1);

- entre les ouverts 1 - et 2 -c.d. et les ouverts $\mathscr{H}$-c.d. (théorème 1.5 );

- entre les ouverts $j$-c.d. et les ouverts $j$-réguliers* $j=1,2$ (théorème 32.2 de [19]).

Soit $\omega \in \mathscr{U}_{c}$. On note :

- $B_{j}$ l'ensemble des points $y \in \partial \omega$ tels que les $j$-potentiels $p_{y}^{j}$ à support $j$-harmonique $\{y\}$ ne soient pas conservés par balayage sur $\complement \omega$ $(j=1,2)$;

- $B$ l'ensemble des points $y \in \partial \omega$ tels que les couples potentiels purs à support biharmonique $\{y\}$ ne soient pas conservés par balayage sur $\complement \omega$.

THÉORÈme 3.3. Les conditions suivantes sont équivalentes:

(i) $\omega$ est un ouvert 1 - et 2-régulier*.

(ii) $\omega$ est un ouvert régulier biharmonique*.

(iii) $B=\emptyset$.

(iv) $B_{1}=\emptyset$ et $B_{2}=\emptyset$.

(v) $\omega$ est $\mathscr{H}$-complètement déterminant.

(vi) $\omega$ est 1 - et 2-complètement déterminant.

Démonstration. (i) $\Leftrightarrow$ (ii). C'est la proposition 3.1.

(v) $\Leftrightarrow$ (vi). C'est le théorème 1.5.

(iv) $\Leftrightarrow$ (vi). Le lemme 24.1 de [19] nous donne la réponse.

(vi) $\Rightarrow$ (ii). C'est la proposition 3.2. 
$(\mathrm{v}) \Rightarrow$ (iii). Grâce à l'hypothèse, $P_{j}^{\mathrm{C} \omega}=p_{j}(j=1,2)$ pour tout couple potentiel $p=\left(p_{1}, p_{2}\right)$, biharmonique dans $\omega$; en particulier, pour $w=\left(w_{y}^{1}, p_{y}^{2}\right)$, couple potentiel pur de support $\{y\} \subset \partial \omega$; donc $W_{1}^{\complement \omega}=w_{y}^{1}, W_{2}^{\complement \omega}=p_{y}^{2}$ et $B=\emptyset$.

(iii) $\Rightarrow(\mathrm{v})$. Comme $B=\emptyset$, alors $\widehat{W}_{1}^{\mathrm{C} \omega}=w_{y}^{1}, \widehat{W}_{2}^{\mathrm{C} \omega}=p_{y}^{2}$ pour $y \in \partial \omega$. Soit maintenant $\mu$ la mesure associée au couple potentiel pur $\left(p_{1}, p_{2}\right)$ biharmonique dans $\omega$ (corollaire 3.13 de [32]); d'où $p_{1}(x)=\int w_{y}^{1}(x) d \mu(y)$ et $p_{2}(x)=\int p_{y}^{2}(x) d \mu(y)$. Donc

$$
\begin{aligned}
\widehat{R}_{p_{1}}^{\mathrm{C} \omega}(x) & =\int p_{1}(z) d \mu_{x}^{\mathrm{C} \omega}(z)=\iint w_{y}^{1}(z) d \mu(y) d \mu_{x}^{\mathrm{C} \omega}(z) \\
& =\int\left(\int w_{y}^{1}(z) d \mu_{x}^{\mathrm{C} \omega}(z)\right) d \mu(y)=\int \widehat{R}_{w_{y}^{1}}^{\mathrm{C} \omega}(x) d \mu(y),
\end{aligned}
$$

avec $\mu_{x}^{\complement \omega}$ la balayée de $\epsilon_{x}$ sur $\complement \omega$ dans l'espace harmonique $\left(\Omega, \mathscr{H}_{1}\right)$.

Par hypothèse,

$$
\begin{aligned}
\widehat{W}_{1}^{\mathrm{C} \omega}(x) & =\int w_{y}^{1}(z) d \mu_{x}^{\mathrm{C} \omega}(z)+\int p_{y}^{2}(z) d \nu_{x}^{\mathrm{C} \omega}(z) \\
& =\widehat{R}_{w_{y}^{1}}^{\mathrm{C} \omega}(x)+\int p_{y}^{2}(z) d \nu_{x}^{\mathrm{C} \omega}(z)=w_{y}^{1}(x)
\end{aligned}
$$

pour $y \in \partial \omega$, où $\left(\mu_{x}^{\mathfrak{C} \omega}, \nu_{x}^{\complement} \omega\right)$ est le couple de mesures balayé du couple $\left(\epsilon_{x}, 0\right)$ (théorème 7.11 de [27]). D'autre part, pour $y \in \complement \bar{\omega}$, grâce au principe du minimum dans $\omega, \widehat{W}_{1}^{\mathrm{C} \omega}=w_{y}^{1}, \widehat{W}_{2}^{\mathrm{C} \omega}=\widehat{R}_{p_{y}^{2}}^{\mathrm{C} \omega}=p_{y}^{2}$ dans $\Omega$. La mesure $\mu$ étant portée par $\complement \omega$, il s'ensuit que

$$
\int \widehat{R}_{w_{y}^{1}}^{\mathrm{C} \omega}(x) d \mu(y)+\iint p_{y}^{2}(z) d \mu(y) d \nu_{x}^{\mathrm{C} \omega}(z)=\int w_{y}^{1}(x) d \mu(y)=p_{1}(x),
$$

d'où

$$
\widehat{P}_{1}^{\complement \omega}(x)=\widehat{R}_{p_{1}}^{\complement \omega}(x)+\int p_{2}(z) d \nu_{x}^{\complement \omega}(z)=p_{1}(x) .
$$

Par conséquent, $\widehat{P}_{1}^{\complement \omega}=p_{1}, \widehat{P}_{2}^{\complement \omega}=\widehat{R}_{p_{2}}^{\complement \omega}=p_{2}$ dans $\Omega$ (voir aussi la remarque 4.3 de [27]); on sait aussi que $\widehat{R}_{p_{2}}^{\mathrm{C \omega}}(x)=\int \widehat{R}_{p_{y}^{2}}^{\mathrm{C} \omega}(x) d \mu(y)$ (théorème 22.4 de [19]).

Comme $\widehat{P}_{j}^{\mathrm{C} \omega} \leq P_{j}^{\mathrm{\complement} \omega} \leq p_{j}$, on aura finalement $P_{j}^{\mathrm{\complement} \omega}=p_{j}(j=1,2)$.

Soit $U \in \mathscr{U}$. Notons ${ }^{a} \mathscr{H}(U)$ (resp. $\left.{ }^{a} \mathscr{H}^{*}(U)\right)$ l'ensemble des couples biharmoniques* (resp. hyperharmoniques*) dans $U$.

Proposition 3.4. L'application $U \mapsto^{a} \mathscr{H}(U)$ définit un faisceau sur $\Omega$ et ${ }^{a} \mathscr{H}(U)$ est un sous-espace vectoriel de $C(U) \times C(U)$.

Démonstration. On remarque que les propositions 2.8 et 2.10 restent encore vraies si l'on suppose seulement que le couple $\left(h_{2}^{*}, h_{1}^{*}\right)$ est défini et biharmonique* dans un voisinage ouvert de chaque point de l'ouvert considéré. Grâce à cette remarque et au théorème 2.13, on démontre la propriété de recollement. La propriété de restriction est évidente. 
Les mesures 1- et 2-harmoniques adjointes définies dans le chapitre 2 nous permettent de construire deux espaces harmoniques (elliptiques) adjoints forts $\left(\Omega,{ }^{a} \mathscr{H}_{1}\right),\left(\Omega,{ }^{a} \mathscr{H}_{2}\right)$ (voir ch. VI de [19]). D'autre part, partant de ces espaces, comme dans la démonstration du lemme 10.11 de [27], on montre qu'il y a identité, dans chaque ouvert $U \in \mathscr{U}$, des fonctions $j$-hyperharmoniques* $(j=1,2)$ et des fonctions de

$$
\begin{aligned}
& { }^{a} \mathscr{H}_{2}^{*}(U)=\left\{v_{2}^{*}:\left(v_{2}^{*}, 0\right) \in{ }^{a} \mathscr{H}^{*}(U)\right\}, \\
& { }^{a} \mathscr{H}_{1}^{*}(U)=\left\{v_{1}^{*}:\left(+\infty, v_{1}^{*}\right) \in{ }^{a} \mathscr{H}^{*}(U)\right\} .
\end{aligned}
$$

On voit donc que :

- L'axiome III (de séparation) est vérifié.

- L'axiome IV (de convergence) est vérifié.

Par conséquent, d'après le théorème 2.13 , il existe une base $\mathscr{B}$ formée d'ouverts réguliers biharmoniques* (axiome II); on a vu aussi que l'axiome de faisceau (axiome I) est vérifié. D'où le

ThÉORÈme 3.5. $\left(\Omega,{ }^{a} \mathscr{H}\right)$ est un espace biharmonique (adjoint).

REMARQUe 3.6. Par construction, les espaces harmoniques adjoints $\left(\Omega,{ }^{a} \mathscr{H}_{1}\right),\left(\Omega,{ }^{a} \mathscr{H}_{2}\right)$ sont les espaces harmoniques associés à l'espace biharmonique $\left(\Omega,{ }^{a} \mathscr{H}\right)$.

4. Couples potentiels adjoints. On conserve les mêmes hypothèses que dans la partie 2 .

On définit comme dans la partie V de [27] les couples potentiels adjoints (ou couples potentiels*).

Soient $\omega \subset \Omega$ ouvert, $x, y \in \Omega,\left(w_{y}, p_{y}^{2}\right)$ le couple de Green de support $\{y\}$ et le couple surharmonique* $\left(w_{x}^{*}, p_{x}^{1 *}\right)$. On désigne par $\left(W_{y}^{\omega},{ }^{2} P_{y}^{\omega}\right)$ la réduite de $\left(w_{y}, p_{y}^{2}\right)$ sur $\omega$, par ${ }^{1} P_{y}^{\omega}$ la réduite de $p_{y}^{1}$ sur $\omega$ et $\operatorname{par}\left(W_{x}^{* \omega},{ }^{1} P_{x}^{* \omega}\right)$ la réduite* du couple $\left(w_{x}^{*}, p_{x}^{1 *}\right)$ sur $\omega$.

Il est connu que $W_{y}^{\omega}(x)=\int w_{y}(u) d \alpha_{x}^{\omega}(u)+\int p_{y}^{2}(u) d \beta_{x}^{\omega}(u)(7.11,7.14$ de [27]), où $\left(\varepsilon_{x}, 0\right)^{\omega}=\left(\alpha_{x}^{\omega}, \beta_{x}^{\omega}\right)$, et ${ }^{1} P_{y}^{\omega}(x)=\int p_{y}^{1}(u) d \alpha_{x}^{\omega}(u)$ (théorème 10.1 de [19]).

Lemme 4.1. Quels que soient l'ouvert $\omega \subset \Omega$ et les points $x$ et $y$ de $\Omega$, on $a W_{y}^{\omega}(x) \geq W_{x}^{* \omega}(y)$.

Démonstration. Le couple

$$
\begin{aligned}
y \mapsto\left(W_{y}^{\omega}(x),{ }^{1} P_{y}^{\omega}(x)\right) & \\
= & \left(\int w_{y}(u) d \alpha_{x}^{\omega}(u)+\int p_{y}^{2}(u) d \beta_{x}^{\omega}(u), \int p_{y}^{1}(u) d \alpha_{x}^{\omega}(u)\right) \\
& =\left(\int w_{y}(u) d \alpha_{x}^{\omega}(u), \int p_{y}^{1}(u) d \alpha_{x}^{\omega}(u)\right)+\left(\int p_{y}^{2}(u) d \beta_{x}^{\omega}(u), 0\right)
\end{aligned}
$$


est la somme de deux couples surharmoniques*, donc il est surharmonique* (lemme 30.1 de [19]; proposition précédente 2.12).

Par ailleurs, si $y \in \omega$, on a $W_{y}^{\omega}(x)=w_{y}(x)$ quel que soit $x \in \Omega$ : en effet, si $U$ est un voisinage ouvert de $y$ tel que $\bar{U} \subset \omega$, alors tout couple surharmonique positif majorant $\left(w_{y}, p_{y}^{2}\right)$ sur $\omega$, donc en particulier sur $\partial U$, le majore sur $\complement U$ (5.11 de [27]); d'où $\left(W_{y}^{\omega}(x),{ }^{2} P_{y}^{\omega}(x)\right)=\left(w_{y}(x), p_{y}^{2}(x)\right)$ pour tout $x \in \Omega$. Grâce à la définition de la réduite ${ }^{*}$, on déduit que $W_{y}^{\omega}(x) \geq$ $W_{x}^{* \omega}(y)$ quels que soient les points $x, y \in \Omega$ : On a déjà montré que $W_{y}^{\omega}(x)=$ $w_{y}(x)$ et l'on sait que, dans l'espace harmonique $\left(\Omega, \mathscr{H}_{1}\right),{ }^{1} P_{y}^{\omega}(x)=p_{y}^{1}(x)$ (lemme 30.2 de [19]). Mais le couple $y \mapsto\left(W_{y}^{\omega}(x),{ }^{1} P_{y}^{\omega}(x)\right)$ est surharmonique* et, d'autre part, il est égal à $\left(w_{y}(x), p_{y}^{1}(x)\right)$ pour $y \in \omega$. D'où le résultat.

Proposition 4.2.

1) Pour tout $x \in \Omega,\left(w_{x}^{*}, p_{x}^{* 1}\right)$ est un couple potentiel ${ }^{*}$ dans $\Omega$, de support $\{x\}$.

2) Pour toute mesure de Radon $\mu$ positive sur $\Omega$, le couple

$$
\left(P_{\mu, 2}^{*}(y), P_{\mu, 1}^{*}(y)\right)=\left(\int w_{x}^{*}(y) d \mu(x), \int p_{x}^{1 *}(y) d \mu(x)\right)
$$

est soit égal à $(+\infty,+\infty)$, soit de la forme $\left(+\infty, q_{1}^{*}\right)$ avec $q_{1}^{*} \in \mathscr{P}_{1}^{*}(\Omega)$, soit un couple potentiel ${ }^{*}$ dans $\Omega$, biharmonique* dans $\Omega \backslash T_{\mu}$. En particulier, si $\mu$ est portée par un compact de $\Omega,\left(P_{\mu, 2}^{*}, P_{\mu, 1}^{*}\right)$ est un couple potentiel* .

Démonstration. 1) En considérant des ouverts $\omega \subset \Omega$ tels que $\complement \omega$ soit compact, on voit que $W_{y}^{\omega}(x) \rightarrow 0$ et ${ }^{2} P_{y}^{\omega}(x) \rightarrow 0$ selon l'ordonné filtrant décroissant formé par ces ouverts, car le plus grand minorant biharmonique du couple potentiel $\left(w_{y}, p_{y}^{2}\right)$ est égal à $(0,0)$. D'autre part, comme $p_{y}^{1}$ est un 1-potentiel, on voit aussi que ${ }^{1} P_{y}^{\omega}(x) \rightarrow 0$ selon le même ordonné filtrant décroissant.

D'après le lemme $4.1, W_{y}^{\omega}(x) \geq W_{x}^{* \omega}(y)$ et, d'après le lemme 30.2 de [19], ${ }^{1} P_{y}^{\omega}(x) \geq{ }^{1} P_{x}^{* \omega}(y)$ quels que soient $x$ et $y$ de $\Omega$. Par conséquent, $W_{x}^{* \omega}(y)$ $\rightarrow 0,{ }^{1} P_{x}^{* \omega}(y) \rightarrow 0$ et $\left(w_{x}^{*}, p_{x}^{1 *}\right)$ est un couple potentiel ${ }^{*}$ dans $\Omega$ (voir aussi proposition 2.12).

2) Grâce à la proposition 2.12 , le couple $\left(P_{\mu, 2}^{*}, P_{\mu, 1}^{*}\right)$ est hyperharmonique* $^{*}$ dans $\Omega$. Comme $P_{\mu, j}^{*}$ est une fonction $j$-hyperharmonique*, ou bien $P_{\mu, j}^{*} \equiv+\infty$ ou bien $P_{\mu, j}^{*}$ est finie en un point $x_{j} \in \Omega, j=1,2[8]$; par suite, le couple $\left(P_{\mu, 2}^{*}, P_{\mu, 1}^{*}\right)$ est ou bien égal à $(+\infty,+\infty)$, ou bien de la forme $\left(+\infty, P_{\mu, 1}^{*}\right)$ avec $P_{\mu, 1}^{*}$ un 1-potentiel* dans $\Omega$ (proposition 30.1 de [19]), ou bien surharmonique* positif dans $\Omega$; dans le dernier cas (proposition 30.1 de [19] $), P_{\mu, j}^{*} \in \mathscr{P}_{j}^{*}(\Omega)(j=1,2)$, donc, grâce à 5.16 de [27], $\left(P_{\mu, 2}^{*}, P_{\mu, 1}^{*}\right) \in$ $\mathscr{P}^{*}(\Omega)$. Enfin, comme dans l'exemple 2.5(2), on montre que ce couple est biharmonique* dans $\Omega \backslash T \mu$. 
Par conséquent, le couple $\left(w_{x}^{*}, p_{x}^{1 *}\right)$ est un couple potentiel* de support biharmonique $\{x\}$.

Remarquons que le raisonnement du lemme 4.1 appliqué au couple potentiel $^{*}\left(w_{x}^{*}, p_{x}^{1 *}\right)$ nous donne l'inégalité $W_{x}^{* \omega}(y) \geq W_{y}^{\omega}(x)$ quels que soient $x$ et $y$ de $\Omega$.

Lemme 4.3. Pour tout ouvert $\omega \subset \Omega$ et $x, y \in \Omega$, on a :

$$
\begin{aligned}
& W_{y}^{\mathrm{\complement} \omega}(x) \geq W_{x}^{* \mathrm{\complement} \omega}(y), \\
& { }^{j} P_{y}^{\mathrm{C} \omega}(x) \geq{ }^{j} P_{x}^{* \mathrm{\complement} \omega}(y) \quad(j=1,2) .
\end{aligned}
$$

Démonstration. On montrera la première inégalité (pour la seconde valable dans l'espace $j$-harmonique, voir lemme 30.2 de [19]).

(i) Soit $y \in \complement \bar{\omega}$; on montre, comme dans la démonstration du lemme 4.1, que $W_{y}^{\mathrm{L} \omega}(x)=w_{y}(x)$ pour tout $x \in \Omega$.

(ii) $y \in \partial \omega$. On distinguera deux cas :

1 er cas : $y$ est 1-polaire. Considérons $\left(v_{1}, v_{2}\right)$ un couple surharmonique dans $\Omega$ majorant $\left(w_{y}, p_{y}^{2}\right)$ dans $\complement \omega$, donc sur $\partial \omega$. On aura

$$
\begin{aligned}
& \liminf _{\omega \ni x \rightarrow z}\left(v_{1}-w_{y}\right)(x) \geq\left(v_{1}-w_{y}\right)(z) \geq 0, \forall z \in \partial \omega \backslash\{y\} ; \\
& \liminf _{\omega \ni x \rightarrow z}\left(v_{2}-p_{y}^{2}\right)(x) \geq\left(v_{2}-p_{y}^{2}\right)(z) \geq 0, \forall z \in \partial \omega \backslash\{y\} \text { ou } \forall z \in \partial \omega
\end{aligned}
$$

respectivement pour y 2-polaire ou non 2-polaire; de l'inégalité (4), on déduit que $v_{2} \geq p_{y}^{2}$ dans $\omega$; d'où $v_{1}-w_{y}$ est 1 -surharmonique dans $\omega$ et, de l'inégalité (3), on a $v_{1} \geq w_{y}$ dans $\omega$. Par conséquent $W_{y}^{\complement \omega}=w_{y}$ et ${ }^{2} P_{y}^{\complement \omega}=p_{y}^{2}$ dans $\Omega$.

2ème cas : $y$ est non 1-polaire. On aura $w_{y}(y)<+\infty$; soit $w_{y}(y)=$ $\lambda>0$. Considérons l'ouvert $G_{\varepsilon}=\left[v_{1}+\varepsilon s_{1}>w_{y}\right]$, où $\left(s_{1}, s_{2}\right)$ est un couple surharmonique positif fini continu, $\left(v_{1}, v_{2}\right)$ un couple comme dans le 1er cas et $\varepsilon>0$; on voit que $G_{\varepsilon} \supset \complement \omega$.

Par conséquent, $W_{y}^{\complement \omega} \leq \inf _{\varepsilon>0} W_{y}^{G_{\varepsilon}} \leq \inf _{\varepsilon>0}\left(v_{1}+\varepsilon s_{1}\right)=v_{1}$ et $W_{y}^{\complement \omega}=$ $\inf _{\varepsilon>0} W_{y}^{G_{\varepsilon}}$. Or, dans un ouvert $U \subset \Omega$, on a $W_{y}^{U}(x)=w_{y}(x)$ pour $y \in U$, $x \in \Omega$, donc $W_{y}^{\complement \omega}(x)=w_{y}(x)$.

En continuant comme dans la démonstration du lemme 4.1, on conclut.

On note qu'en appliquant le raisonnement précédent au couple potentiel $\left(w_{x}^{*}, p_{x}^{1 *}\right)$, on trouve l'inégalité $W_{x}^{* \complement} \omega(y) \geq W_{y}^{\complement \omega}(x)$ quels que soient $x$ et $y$ dans $\Omega$.

Dorénavant, on suppose la proportionnalité des $j$-potentiels adjoints de support ponctuel $(j=1,2)$.

Proposition 4.4. Pour tout $\omega \in \mathscr{U}_{c}$, les trois mesures biharmoniques** $\lambda_{x}^{\prime}, \mu_{x}^{\prime}, \nu_{x}^{\prime}$ coïncident respectivement avec les mesures $\lambda_{x}^{\omega}, \mu_{x}^{\omega}, \nu_{x}^{\omega}$. 
Démonstration. Soit $x \in \omega$. Alors ${ }^{1} \widehat{P}_{x}^{* \mathrm{C} \omega}(y)=\int p_{u}^{1 *}(y) d \mu_{x}^{\prime}(u)$ est un 1-potentiel* de mesure $\mu_{x}^{\prime}$ portée par le compact $\partial \omega$. Le couple potentiel*

$$
\left(\widehat{W}_{x}^{* \mathrm{C} \omega}(y),{ }^{1} \widehat{P}_{x}^{* \mathrm{C} \omega}(y)\right)=\left(\int w_{u}^{*}(y) d \mu_{x}^{\prime}(u), \int p_{u}^{1 *}(y) d \mu_{x}^{\prime}(u)\right)+\left(s_{2}^{*}, 0\right)
$$

où $s_{2}^{*}$ est un 2-potentiel*; donc, $s_{2}^{*}(y)=\int p_{u}^{* 2}(y) d \nu_{x}^{\prime}(u)(2.2,3.14$ de [32]). Or, $\mu_{x}^{\prime}$ est la mesure 1-harmonique $\mu_{x}^{\omega}$, car, dans $\omega,{ }^{1} P_{x}^{* \mathrm{C \omega} \omega}(y)={ }^{1} P_{y}^{\complement \omega}(x)=$ $H_{p_{y}^{1}}^{\omega}(x)$ (voir [19]). On voit aussi que $\lambda_{x}^{\prime}$ est la mesure 2-harmonique $\lambda_{x}^{\omega}$.

On a donc

$$
\widehat{W}_{x}^{* \mathrm{C} \omega}(y)=\int w_{u}^{*}(y) d \mu_{x}^{\prime}(u)+\int p_{u}^{* 2}(y) d \nu_{x}^{\prime}(u)
$$

D'autre part,

$$
\widehat{W}_{y}^{\complement \omega}(x)=\int w_{y}(u) d \alpha_{x}^{\complement \omega}(u)+\int p_{y}^{2}(u) d \beta_{x}^{\complement \omega}(u),
$$

et $\alpha_{x}^{\mathrm{C} \omega}=\mu_{x}^{\omega}, \beta_{x}^{\mathrm{C} \omega}=\nu_{x}^{\omega}$ si $x \in \omega$ (voir partie VII de [27]).

Dans $\omega, W_{y}^{\complement \omega}=\widehat{W}_{y}^{\complement \omega}$ et $W_{x}^{* \mathrm{C} \omega}=\widehat{W}_{x}^{* \mathrm{C} \omega}$.

Comme $W_{y}^{\mathrm{C} \omega}(x)=W_{x}^{* \mathrm{C} \omega}(y)$ quels que soient $x, y \in \Omega$ (lemme 4.3), on aura dans $\omega$

$$
\begin{aligned}
W_{x}^{* \mathrm{C} \omega}(y) & =\int w_{y}(u) d \mu_{x}^{\prime}(u)+\int p_{y}^{2}(u) d \nu_{x}^{\prime}(u) \\
& =\int w_{y}(u) d \mu_{x}^{\omega}(u)+\int p_{y}^{2}(u) d \nu_{x}^{\omega}(u)=W_{y}^{\mathrm{C} \omega}(x) .
\end{aligned}
$$

Comme $\mu_{x}^{\prime}=\mu_{x}^{\omega}$, on a $\int p_{y}^{2}(u) d \nu_{x}^{\prime}(u)=\int p_{y}^{2}(u) d \nu_{x}^{\omega}(u)$, d'où $\nu_{x}^{\prime}=\nu_{x}^{\omega}$.

Proposition 4.5. Le couple potentiel $\left(w_{x}^{*}, p_{x}^{1 *}\right)$ est un couple adjoint pur pour tout $x \in \Omega$.

Démonstration. Soit un point $x \in \Omega$ et le couple $\left(w_{x}^{*}, p_{x}^{1 *}\right)$. Il y a deux possibilités : 1) ce couple est adjoint pur; donc, pour ce point $x \in \Omega$, on a le résultat. 2) Ce couple n'est pas adjoint pur et l'on aura $\left(w_{x}^{*}, p_{x}^{1 *}\right)=$ $\left(u_{x}^{*}, p_{x}^{1 *}\right)+\left(p_{x}^{2 *}, 0\right)$, où $\left(u_{x}^{*}, p_{x}^{1 *}\right)$ est le couple pur adjoint associé et $p_{x}^{2 *}$ un 2-potentiel adjoint (3.8 de [32]).

Soit

$$
D=\left\{x \in \Omega:\left(w_{x}^{*}, p_{x}^{1 *}\right) \text { n'est pas pur* }\right\}
$$

et soit $x_{0} \in D$. On a

$$
w_{x_{0}}^{*}(y)=u_{x_{0}}^{*}(y)+p_{x_{0}}^{2 *}(y) \quad(\forall y \in \Omega) .
$$

On a vu que l'espace biharmonique** coïncide avec l'espace biharmonique initial (proposition 4.4). Donc, au couple $\left(u_{x}^{*}, p_{x}^{1 *}\right)$ correspond le couple $\left(u_{y}(x), p_{y}^{2}(x)\right)$ dans l'espace biharmonique initial et l'on montre, comme dans la démonstration de la proposition 4.2, que ce couple est un couple potentiel dans $\Omega$ de support $\{y\}$. De la relation (5), il s'ensuit que

$$
w_{y}\left(x_{0}\right)>u_{y}\left(x_{0}\right) \text {. }
$$


Le couple $\left(u_{y}, p_{y}^{2}\right)$ est ou bien un couple pur et, dans ce cas-là, $u_{y}=w_{y}$, car le couple $\left(w_{y}, p_{y}^{2}\right)$ est un couple pur, d'où contradiction à l'inégalité précédente; ou bien, il n'est pas pur et alors

$$
\left(u_{y}, p_{y}^{2}\right)=\left(w_{y}, p_{y}^{2}\right)+\left(p_{y}^{1}, 0\right) \quad(\text { voir } 3.8 \text { de [32] }) .
$$

Donc, $u_{y}>w_{y}$, d'où encore contradiction.

Le point $x$ étant quelconque, on conclut que $D=\emptyset$; par conséquent tous les couples $\left(w_{x}^{*}, p_{x}^{1 *}\right)$ sont purs.

5. Applications. Soit $L_{j}(j=1,2)$ un opérateur différentiel linéaire du second ordre elliptique, avec des coefficients assez réguliers, défini dans un domaine $\Omega$ de $\mathbb{R}^{n}(n \geq 2)$.

Considérons l'espace biharmonique des solutions $\left(u_{1}, u_{2}\right)$ du système

$$
L_{1} u_{1}=-u_{2}, \quad L_{2} u_{2}=0
$$

dans $\Omega$ et supposons qu'il existe un couple potentiel strictement positif; il existe donc un $L_{j}$-potentiel $>0(j=1,2)$ (partie XII de [27]).

On sait (chapitre VII de [19]) que, dans un $L$-espace harmonique elliptique avec potentiel $>0$, les $L$-potentiels dans $\Omega$ de support $\{y\}$ sont proportionnels et il existe un $L$-potentiel $P_{y}$ de support $\{y\}$ tel que $P_{y}(x) \sim H_{y}(x)$ quand $x \rightarrow y$, et que, pour tout $x \in \Omega$, la fonction $y \mapsto P_{y}(x)$, continue dans $\Omega \backslash\{x\}$, est le potentiel de singularité $x$ pour l'opérateur adjoint $L^{*}$. (Ce résultat s'applique aux opérateurs $L_{j}$ et $L_{j}^{*}$.)

On note $P_{j}(x, y)=P_{y}^{j}(x)$ et $P_{j}^{*}(x, y)=P_{y}^{* j}(x)$.

Dans notre espace biharmonique, soit $P_{y}^{2}$ un $L_{2}$-potentiel et $\left(W_{y}, P_{y}^{2}\right)$ le couple potentiel pur associé.

A) À l'aide de $\left(W_{y}, P_{y}^{2}\right)$ et d'un $L_{1}$-potentiel (choisi) $P_{y}^{1}$, aux couples $L_{2} L_{1}$-biharmoniques, on associe le système de couples $L_{2} L_{1}$-biharmoniques adjoints. Comme dans le cas harmonique, on montre que les couples adjoints forment un espace biharmonique et que les couples adjoints sont des solutions du système $L_{2}^{*} h_{2}=-h_{1}, L_{1}^{*} h_{1}=0$ (voir remarque précédente 2.4, chapitre VII de [19], partie XII de [27] et p. 13-21 de [24]).

B) On donnera maintenant l'expression des couples potentiels purs. Soit $\omega$ un ouvert relativement compact de notre espace avec frontière régulière, où les fonctions de Green pour les opérateurs $L_{1}, L_{2}$ (et $L_{1}^{*}, L_{2}^{*}$ ) existent, et soit $G_{1}^{\omega}, G_{2}^{\omega}$ les fonctions de Green respectives (voir [5], 7], [11], [14]).

On voit, en appliquant le raisonnement du théorème 17 de [28, que $p^{\omega}(x, y)=\int G_{1}^{\omega}(x, z) G_{2}^{\omega}(z, y) d z$ est, dans $\omega$, le potentiel pur associé à $G_{2}^{\omega}$.

On considère une suite $\left(\omega_{k}\right)$ croissante de tels ouverts et telle que $\overline{\omega_{k}} \subset \omega_{k+1}, k=1,2, \ldots$, et $\omega_{k} \nearrow \mathbb{R}^{n}$. 
Avec des hypothèses appropriées (voir partie XII de [27], p. 49-59 de [24] et remarque 2.4), en passant à la limite pour $k \rightarrow \infty$, on trouve que

$$
w_{y}(x)=\int P_{1}(x, z) P_{2}(z, y) d z .
$$

Procédure analogue pour exprimer le $w_{x}^{*}$.

C) Les ensembles polaires pour l'opérateur composé et son adjoint sont les mêmes et ils sont identiques aux ensembles $\Delta$-polaires; il y a aussi identité entre les points-frontières $L_{2} L_{1}$-réguliers, $\left(L_{2} L_{1}\right)^{*}$-réguliers et $\Delta$ réguliers (voir [29] et chapitre VII de [19]).

\section{Références}

[1] H. Bauer, Harmonic spaces and associated Markov processes, dans : Potential Theory, C.I.M.E., Ed. Cremonese, Roma, 1970, 23-67.

[2] J. Bliedtner and W. Hansen, Potential Theory, Springer, 1986.

[3] N. Boboc, Gh. Bucur and A. Cornea, H-cones and potential theory, Ann. Inst. Fourier (Grenoble) 25 (1975), no. 3-4, 71-108.

[4] - , - - - Order and Convexity in Potential Theory, Lecture Notes in Math. 853, Springer, 1981.

[5] J. M. Bony, Principe du maximum, inégalité de Harnack et unicité du problème de Cauchy pour les opérateurs elliptiques dégénérés, Ann. Inst. Fourier (Grenoble) 19 (1969), no. 1, 277-304.

[6] N. Bouleau, Espaces biharmoniques, systèmes d'équations différentiels et couplage de processus de Markov, J. Math. Pures Appl. 59 (1980), 187-240.

[7] M. Brelot, Éléments de la théorie classique du potentiel, 4e éd., Centre de documentation universitaire, Paris, 1969.

[8] —, Lectures on Potential Theory, Tata Inst., Bombay, 1960.

[9] —, Axiomatique des fonctions harmoniques, Presses Univ. de Montréal, 1966.

[10] C. Constantinescu and A. Cornea, Potential Theory on Harmonic Spaces, Springer, 1972.

[11] R. Courant and D. Hilbert, Methods of Mathematical Physics, Vol. II, Interscience, 1966.

[12] J. Dieudonné, Éléments d'Analyse, t. II, Gauthier-Villars, Paris, 1969.

[13] J. L. Doob, Classical Potential Theory and its Probabilistic Counterpart, Springer, 1984.

[14] Y. V. Egorov and M. A. Shubin, Foundations of the Classical Theory of Partial Differential Equations, Springer, 1998.

[15] D. Feyel et A. de la Pradelle, Cônes en dualité. Applications aux fonctions de Green, dans : Séminaire de théorie du potentiel, Lecture Notes in Math. 563, Springer, 1976, $62-99$.

[16] —, 一, Dualité des quasi-résolvantes de Ray, dans : Séminaire de théorie du potentiel, Lecture Notes in Math. 713, Springer, 1979, 67-88.

[17] D. Gilbarg and N. Trudinger, Elliptic Partial Differential Equations of Second Order, Springer, 1983.

[18] E. Goursat, Cours d'analyse mathématique, t. III, Gauthier-Villars, Paris, 1927.

[19] R. M. Hervé, Recherches axiomatiques sur la théorie des fonctions surharmoniques et du potentiel, Ann. Inst. Fourier (Grenoble) 12 (1962), 415-571. 
[20] L. Hörmander, Linear Partial Differential Operators, Springer, 1963.

[21] T. Ikegami, Duality on harmonic spaces, Osaka J. Math. 28 (1991), 93-116.

[22] F.-Y. Maeda, Duality of two balayage-space structures and Dirichlet integrals on balayage spaces, Potential Anal. 4 (1995), 595-613.

[23] P. A. Meyer, Brelot's axiomatic theory of the Dirichlet problem and Hunt's theory, Ann. Inst. Fourier (Grenoble) 13 (1963), no. 2, 357-372.

[24] C. Miranda, Equazioni alle derivate parziali di tipo ellittico, Ergeb. Math. Grenzgeb. 2, Springer, 1955.

[25] G. Mokobodzki, Pseudo-quotient de deux mesures par rapport à un cône de potentiels. Applications à la dualité, dans : Séminaire de probabilité VI (Strasbourg, 1970/1971), Lecture Notes in Math. 258, Springer, 1972, 173-176.

[26] D. Sibony, Cônes de fonctions et potentiels, Cours de 3ème cycle à la Faculté des Sciences de Paris, 1967-1968.

[27] E. P. Smyrnelis, Axiomatique des fonctions biharmoniques, Ann. Inst. Fourier (Grenoble) 25 (1975), no. 1, 35-97 et 26 (1976), no. 3, 1-47.

[28] —, Sur les fonctions hyperharmoniques d'ordre 2, dans : Séminaire de théorie du potentiel (Paris, 1976/1977), Lecture Notes in Math. 681, Springer, 1978, 277-294.

[29] —, Polarité et effilement dans les espaces biharmoniques, dans : Séminaire de théorie du potentiel (Paris, 1976/1977), Lecture Notes in Math. 681, Springer, 1978, 252276.

[30] —, Axiome de domination dans les espaces biharmoniques, dans : Séminaire de théorie du potentiel (Paris), Lecture Notes in Math. 1061, Springer, 1984, 271-281.

[31] - Une propriété de moyenne des fonctions biharmoniques, Bull. Sci. Math. (2) 109 (1985), 103-111, 446.

[32] - Représentation intégrale dans les espaces biharmoniques, Acad. Roy. Belg. Bull. Cl. Sci. 71 (1985), 383-394.

[33] -, Couples de Green, ibid. 13 (2002), 319-326.

[34] J. C. Taylor, Duality and the Martin compactification, Ann. Inst. Fourier (Grenoble) 22 (1972), no. 3, 95-130.

Emmanuel P. Smyrnelis

Department of Mathematics

University of Athens

Athens, Greece

E-mail: smyrnel@math.uoa.gr

Received 27.4.2009

and in final form 8.11.2009 
